\title{
Diagnostic utility of trophoblastic cell surface antigen 2 immunohistochemical expression in papillary thyroid carcinoma
}

\author{
Nesreen H. Hafez', Manal Z. Shalaby'2, Mohamed M. Ahmed ${ }^{3}$ \\ ${ }^{I}$ Department of Pathology, National Cancer Institute, Cairo University, Cairo, Egypt. \\ ${ }^{2}$ Department of Medicine, National Research Center, Egypt. \\ ${ }^{3}$ Department of Reproductive Health and Family Planning Research, National Research Center, Egypt.
}

\author{
Keywords: \\ Cytology; \\ Histopathology; \\ Immunohistochemistry; \\ Papillary thyroid \\ carcinoma; \\ TROP-2;
}

\begin{abstract}
Background: Although nuclear criteria are considered to be characteristic for papillary thyroid carcinoma, some cases do raise controversy as being papillary thyroid carcinoma or non-papillary thyroid carcinoma due to unclear diagnostic nuclear features. Immunohistochemical markers can help in identifying PTC. Trophoblastic cell surface antigen 2 (TROP-2) is a novel marker that is recently applied on thyroid. The aim of this study was to evaluate TROP-2 expression and diagnostic utility in PTC compared to other thyroid nodules with follicular pattern on tissue specimens and cell block sections.
\end{abstract}

Materials and Methods: This retrospective study was carried out at National Cancer Institute, Cairo University between January 2010 and January 2015. Immunohistochemical evaluation of TROP-2 was applied on archival 110 surgically excised and 66 cytological cell blocks of PTC and other follicular thyroid nodules. Membranous staining, with or without cytoplasm, was considered positive.

Results: Among excised specimens, $85.1 \%$ of PTC demonstrated TROP-2 staining. The majority revealed diffuse staining. No significant staining difference was reported between studied classic and non classic variants. TROP-2 sensitivity and were $85.1 \%$ and $94.4 \%$. Among cytological materials, all PTC were positive except two (false negative) and only two follicular adenomas were positive (false positive). TROP-2 sensitivity and specificity were $93.8 \%$ and $94.1 \%$. None of nodular hyperplasia or adjacent normal thyroid tissues showed TROP-2 immunoreactivity either on histopathological or cytological sections.

Conclusion: TROP-2 positivity was significantly higher in PTC compared to all other studied non PTC lesion in both surgical pathology specimens and cytological materials. No reported significant staining difference among studied PTC variants.

\section{Correspondence:}

Dr. Nesreen Hassan Hafez

Department of Pathology

National Cancer Institute, Cairo University

ORCID ID number: orcid.org/0000-0001-6608-3230

E-mail:nesreennci@hotmail.com

Received : August 20th 2017; Accepted : November 6th 2017; Published : March 21st 2018

Citation: Nesreen HH, Manal ZS, Mohamed MA. Diagnostic utility of trophoblastic cell

surface antigen 2 immunohistochemical expression in papillary thyroid carcinoma. J Pathol Nep 2018;8:1235-43. Doi: 10.3126/jpn.v8i1.19442

Copyright: This is an open-access article distributed under the terms of the Creative Commons Attribution 4.0 International License, which permits unrestricted use, distribution, and reproduction in any medium, provided the original author and source are credited.

\section{INTRODUCTION}

Thyroid nodules are a common clinical problem. It is estimated that $3 \%$ to $7 \%$ of the world's population have a palpable nodule, and the prevalence may increase to more than $70 \%$ if patients are screened by high-resolution ultrasound. ${ }^{1}$ The major concern in such patients is to rule out the possibility of malignancy. Approximately 5\% of detected thyroid nodules are malignant. Although thyroid cancer account for only $1 \%$ of overall human cancer, they represent the most common endocrine related cancer. ${ }^{2}$ It is 
traditionally believed that solitary thyroid nodules are more likely to be neoplastic than multiple nodules. ${ }^{3}$ However, in recent past, various studies have reported a rising incidence of malignancy even in multinodular goiter. ${ }^{4}$ Differentiated thyroid cancer, which includes papillary and follicular cancer, comprises the vast majority ( $>90 \%)$ of all thyroid cancers. ${ }^{1}$

Papillary thyroid carcinoma (PTC) is the commonest thyroid cancer accounting approximately $85 \%$ of all thyroid cancers. Pathological diagnosis in both cytological and histological materials is straightforward in most cases and it is considered the gold standard for diagnosis. Proper diagnosis of PTC is crucial as it affects patients' clinical management and prognosis. ${ }^{5}$ However, pathologists may be confronted with neoplasm exhibiting unclear nuclear diagnostic criteria for PTC that makes diagnosis difficult, especially in specimens with low cellularity. Diagnostic problem may also arise when an encapsulated nodule with a follicular growth pattern exhibits some nuclear criteria for PTC and so distinguishing follicular adenoma (FA) or follicular carcinoma (FC) from encapsulated follicular variant of PTC (FVPTC) becomes difficult. Other thyroid lesions, benign papillary hyperplasia, may have papillary patterns with focal PTC-like nuclear features, which cause diagnostic difficulties. Unfortunately such controversy exists even between expert thyroid pathologists. ${ }^{6}$ A growing number of some promising immunohistochemical (IHC) and molecular markers for differentiating PTC from other thyroid follicular lesions have emerged. ${ }^{7}$ Molecular methods required expensive equipment and rigorous quality control. Immunostaining is simple, relatively inexpensive, and routinely performed in most pathology laboratories. IHC markers included cytokeratin-19 (CK-19), galectin-3 (GAL3), and hector battifora mesothelial antigen-1 (HBME1) have been shown to be useful in reducing the incidence of difficult diagnoses. They are recommended to be used as a panel. ${ }^{8}$

Trophoblastic cell surface antigen 2 (TROP-2) is a novel marker that is applied on thyroid. It is a transmembrane glycoprotein encoded by tumor-associated calcium signal transducer2 gene (TACSTD2) of chromosome $1 \mathrm{p} 32$. TROP-2 was initially detected in human trophoblasts and choriocarcinoma cell lines. It participates in regulation of intracellular concentration of calcium ions. It was found to be associated with regulation of cyclin D1 and protein kinase $\mathrm{C}$ levels, which activate the mitogen-activated protein kinase/ extracellular signal-regulated kinase pathway to increase expression of cyclin D1 and cyclin E. ${ }^{9}$ It has been shown to be associated with tumor development and progression in a variety of epithelial carcinomas compared with corresponding normal tissues. ${ }^{10}$ In normal tissues, its expression was reported as low or lacking. Many studies have demonstrated that TROP-2 overexpression is associated with tumor aggressiveness and poor prognosis, so it has been studied as a prognostic marker. ${ }^{11}$ Recently, TROP-2 has been explored as an immunotherapeutic target therapy in human cancer. Anti-Trop-2 monoclonal antibodies have been shown to kill uterine serous papillary carcinoma cells in vitro. ${ }^{10,12}$

Recently, few studies have investigated the role of TROP2 in diagnosing PTC and have reported that TROP-2 is a promising marker for identifying PTC. ${ }^{12}$ These studies urged us to evaluate TROP-2 expression and its diagnostic utility on tissue histopathological specimens and cell block cytological sections of PTC cases as well as other thyroid nodules with follicular pattern including hyperplastic nodules, FA and follicular carcinoma.

\section{MATERIALS AND METHODS}

This retrospective study included 110 surgically excised, formalin-fixed, paraffin embedded blocks of PTC specimens and other thyroid nodules with follicular pattern as well as 66 cytological cell blocks obtained from other thyroid nodules that were aspirated preoperatively. All blocks were retrieved from the archives of the Pathology Department, National Cancer Institute, Cairo University between January 2010 and January 2015. Cases were selected from pathological electronic database. Permission was obtained from institutional review committee.

All histopathological cases were stained with routine hematoxylin-eosin staining and diagnosed and classified according to World Health Organization classification. ${ }^{13}$ All cytological materials were stained with Papanicolaou stain and classified according to Bethesda system for reporting thyroid cytopathology. ${ }^{14}$

Inclusion criteria for surgical specimens included patients with any age and sex that underwent resection or biopsy for thyroid nodules with final histopathological unequivocal diagnosis of PTC, hyperplastic nodules, FA or FC cases. Cases with equivocal or indefinite histopathological diagnosis were excluded from the study. Inclusion criteria for cytological materials involved cytological diagnosis of PTC cases or other follicular lesions with final surgically proven diagnosis as well as availability of adequate cell block for serial cutting and staining. Patients with other thyroid lesions or malignancies were excluded. None of the patients received radiotherapy, chemotherapy or endocrine therapy prior to surgery or cytology.

Clinicopathological information was retrieved from pathology records. All patients underwent full laboratory work-up, chest X-ray and CT scan of the neck and upper mediastinum. Fibro-optic endoscopy was ordered for patients presenting with hoarseness of voice and other upper respiratory symptoms. 


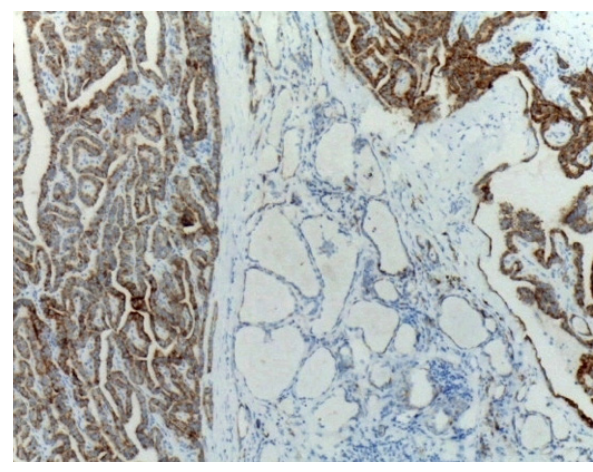

Figure 1: Immunohistochemical expression of TROP-2 in classic PTC reveals diffuse positivity in tumor cells and negative in adjacent residual thyroid tissue (Immunoperoxidase; X100)

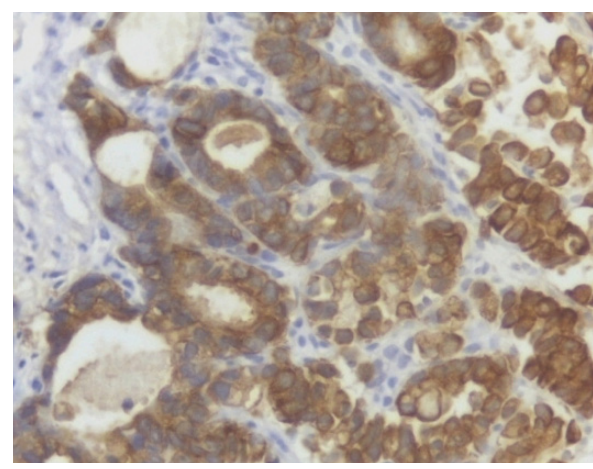

Figure 3: Immunohistochemical TROP-2 expression in FC case reveals diffuse positivity in tumor cells (Immunoperoxidase; X400).

The archival histopathological and cytological slides were reviewed to confirm the diagnosis and to choose the appropriate paraffin embedded tissue and cytological cell blocks that contain the most representative and wellpreserved tumor areas for sectioning and IHC staining. For each case; one serial section, of $4 \mu \mathrm{m}$ thickness were cut by the microtome then mounted onto positively charged slides.

The staining process was strictly performed according to streptavidin-biotin-peroxidase complex method according to the manufacturer's protocol using BenchMark XT automated slide stainer (a product of Ventana Medical Systems). Paraffin sections were deparaffinized by xylene, rehydrated by a graded series of alcohol. Sections were incubated for $5 \mathrm{~min}$ with $0.3 \%$ hydrogen peroxide at room temperature to block endogenous peroxidase activity. Heatbased antigen retrieval was performed to obtain optimal results. Sections were treated with $5 \%$ bovine serum albumin to block non-specific staining. The slides were incubated with the primary anti- TROP-2 antibody (mouse monoclonal IgG1, clone F-5, dilution 1:50, Cat sc-376181, Santa Cruz, CA). 3',3' diaminobenzidine hydrochloride was used as a chromogen and hematoxylin as a counterstain.

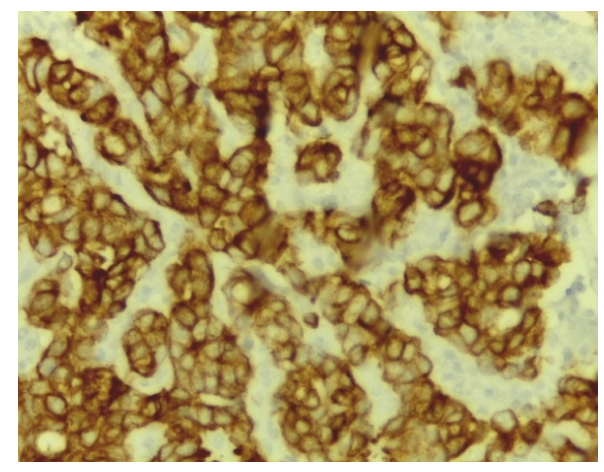

Figure 2: Immunohistochemical expression of TROP-2 in classic PTC reveals diffuse positivity in tumor cells (Immunoperoxidase; X400).

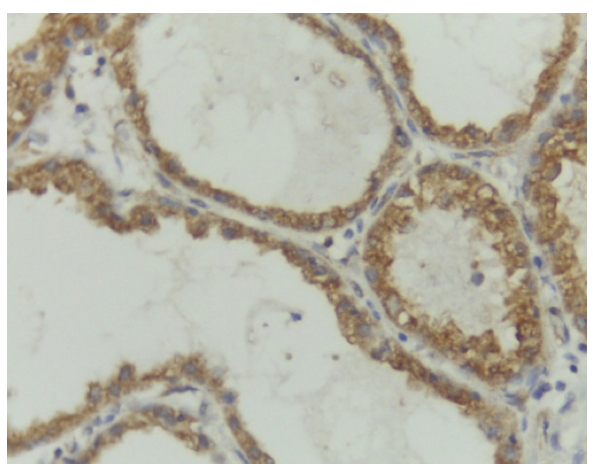

Figure 4: mmunohistochemical expression of TROP-2 in nodular hyperplasia reveals cytoplasmic staining with no membranous staining pattern, negative (Immunoperoxidase; X400).

Appropriate positive and negative controls were included in each IHC run. Negative controls were prepared by replacing the primary antibody with Phosphate Buffer Saline (PBS). Normal human skin was used as positive control. TROP-2 was localized in the epidermis, but cells of the basal layer were negative for immunohisto chemistry.

Immunohistochemistry and ICC data were assessed by the pathologist using Olympus binocular microscope. The cells were regarded as positive when immunoreactivity was clearly observed in their cell membranes with or without cytoplasm. Only cytoplasmic or nuclear staining for TROP2 or colloid staining in absence of follicular epithelium membranous staining was considered to be non-specific. According to Liu et al..$^{15}$ staining distribution was recorded as negative if no staining or $<5 \%$ stained tumor cells, $1+$ if $5 \%$ to $25 \%$ of cells were stained, $2+$ if $26 \%$ to $50 \%$ of cells were stained, $3+$ if $51 \%$ to $75 \%$ of cells were stained, or $4+$ if $>75 \%$ of cells were stained. Scores $1+, 2+, 3+$ and $4+$, regardless of staining intensity, were considered positive. A diffuse staining pattern was referred to score $3+$ or $4+$. The percentage of positive cells was confirmed by a second independent examination, blinded to the initial score. The 
Table 1: Cytological diagnoses and its comparison with corresponding histopathological diagnoses.

\begin{tabular}{|c|c|c|}
\hline $\begin{array}{l}\text { Cytological } \\
\text { Diagnosis }\end{array}$ & Number & Histopathological Follow up \\
\hline PTC & 25 & PTC, classic variant $(n=25)$ \\
\hline Suspicious & 5 & $\begin{array}{l}\text { PTC, classic variant }(n=4) \\
\text { PTC, microcarcinoma }(n=1)\end{array}$ \\
\hline FN & 15 & $\begin{array}{l}\text { FA }(n=12) \\
\text { FC }(n=1) \\
\text { Hyperplastic nodule }(n=2)\end{array}$ \\
\hline FLUS & 11 & $\begin{array}{l}\text { FA }(n=9) \\
\text { PTC, classic variant }(n=2)\end{array}$ \\
\hline $\begin{array}{l}\text { Nodular } \\
\text { Hyperplasia }\end{array}$ & 10 & Hyperplastic nodule $(\mathrm{n}=10)$ \\
\hline Total & 66 & \\
\hline
\end{tabular}

PTC: papillary thyroid carcinoma; FC: follicular carcinoma; FA: follicular adenoma; FN: follicular neoplasm; FLUS:

follicular lesion of undetermined significance.

readings gave nearly similar results.

Cases were studied in two groups, PTC group and non PTC group. Statistical analyses were performed using GraphPad Prism version 7.00 for window (GraphPad Software, La Jolla, CA, USA: www.Graphpad.com). Sensitivity for the presence of PTC, specificity for absence of PTC, positive predictive value (PPV) for the probability that the patient with positive test has, in fact, the disease in question, negative predictive value (NPV) for the probability of a patient with a negative test not having the disease in question and accuracy were calculated. The statistical analyses were performed with Fisher's exact and Chi-square analysis to evaluate associations of categorical data. Continuous data were presented with mean \pm standard deviation (SD) using $\mathrm{t}$ test for comparison. $\mathrm{P}$ values equal or less than 0.05 was used to determine the statistically significance.

\section{RESULTS}

A total of 110 surgically excised thyroid specimens and 66 cytological cell block materials were included in the current study. The surgically excised specimens included 29 males, and 81 females, with a male to female ratio of
1:2.8. The age ranged from 22 to 71 years, with the mean age being $49.1 \pm 7.1$ years. Fifty patients $(45.4 \%)$ presented with nodules in right thyroid lobe, forty one cases $(37.3 \%)$ presented with nodules in left lobe while nineteen patients $(17.3 \%)$ presented with isthmus nodules.

The selected pathological cases included unequivocal diagnosis of 74 PTC (67.3\%), 14 FA (12.7\%), 7 FC (6.4\%) and 15 cases of hyperplastic nodules $(13.6 \%)$. PTC cases were further classified into 61 classic variant PTC, 11 FVPTC and 2 tall cell variant PTC. Follicular carcinoma cases were minimally invasive in 6 cases and widely invasive in 1 case. FA cases included 4 Hurthle cell subtype. In addition, adjacent residual normal thyroid tissue was included with 24 primary tumors in the same studied sections. Cases were studied in two groups, PTC group and non PTC group.

Patients that underwent fine needle aspiration cytology for thyroid follicular lesions comprising of 15 males and 51 females had a mean age of $36.3 \pm 12.0$ years (ranged from 19 to 62 years). Male to female ratio was 1:3.4. The selected cytological cases included 25 PTC, 5 suspicious for malignancy, 15 follicular neoplasm (FN), 11 follicular lesions of undetermined significance (FLUS), and 10 cases were of nodular hyperplasia. All cytological cases had confirmatory diagnoses on surgical histopathological followup and adequate cell blocks. Cytological diagnoses of 66 included cell block sections compared with corresponding histopathological diagnoses were shown in Table 1.

\section{TROP-2 expression in excised thyroid tissue specimens:}

Among PTC specimens, 63 out of 74 cases (85.1\%) demonstrated TROP-2 staining (fig. 1 and fig. 2). Regarding staining distribution, the majority of PTC positive cases, 48 cases, revealed diffuse staining $(3+$ and $4+)$. Eight cases showed moderate staining (2+). Seven cases showed focal staining (1+). Stromal cells were negative for TROP-2. None of PTC cases showed only cytoplasmic or nuclear staining. TROP 2 expression of the studied excised thyroid specimens was summarized in Table 2. In contrast to PTC, only one out of seven FC cases (14.3\%) was TROP-2 positive (fig.3).

Table 2: TROP-2 expression in histopathological excised thyroid specimens

\begin{tabular}{|c|c|c|c|c|c|c|c|}
\hline \multirow{3}{*}{ Histopathologic Diagnosis } & \multirow{3}{*}{ Number $(n=110)$} & \multicolumn{6}{|c|}{ TROP-2: human trophoblast cell-surface marker } \\
\hline & & \multirow[t]{2}{*}{ Negative No. (\%) } & \multirow[t]{2}{*}{ Positive No. (\%) } & \multicolumn{4}{|c|}{ Distribution score } \\
\hline & & & & $1+$ & $2+$ & $3+$ & $4+$ \\
\hline PTC & 74 & $11(14.9)$ & $63(85.1)$ & 7 & 8 & 20 & 28 \\
\hline Non PTC Lesions & 36 & & & & & & \\
\hline - FA & 14 & $13(92.9)$ & $1(7.1)$ & 1 & 0 & 0 & 0 \\
\hline - FC & 7 & $6(85.7)$ & $1(14.3)$ & 0 & 0 & 1 & 0 \\
\hline - Hyperplastic Nodule & 15 & $15(100)$ & 0 & 0 & 0 & 0 & 0 \\
\hline
\end{tabular}

PTC: papillary thyroid carcinoma; FC: follicular carcinoma; FA: follicular adenoma; 
Table 3: TROP-2 expression in PTC histopathological variants.

\begin{tabular}{lcccccc}
\hline \multicolumn{1}{c}{} & \multicolumn{5}{c}{ TROP-2 Expression } \\
\hline Histopathological PTC Variants & Number $(\mathbf{n = 1 1 0 )}$ & Positive No. (\%) & \multicolumn{4}{c}{ Distribution score } \\
\hline Classic variant & & & $\mathbf{1 +}$ & $\mathbf{2 +}$ & $\mathbf{3 +}$ & $\mathbf{4 +}$ \\
\hline Follicular variant & 61 & $53(86.9)$ & 6 & 8 & 15 & 24 \\
\hline Tall variant & 11 & $8(72.7)$ & 1 & 0 & 4 & 3 \\
\hline Total & 2 & $2(100)$ & 0 & 0 & 1 & 1 \\
\hline
\end{tabular}

PTC: papillary thyroid carcinoma; TROP-2: human trophoblast cell-surface marker

This positive case showed staining pattern in about $60 \%$ of examined cells $(3+)$. Another FA case out of fourteen cases $(7.1 \%)$ revealed TROP-2 staining. This case showed positivity in about $10 \%$ of examined cells $(1+)$. All samples of nodular hyperplasia and adjacent normal thyroid tissues were negative. TROP-2 positivity as well as staining distributions was significantly higher in PTC compared to all other studied neoplastic and non-neoplastic thyroid lesions ( $p$ value $<0.001$ ). Two FA and one nodular hyperplasia cases revealed only cytoplasmic staining and they were considered negative (fig. 4).

TROP-2 expression in different studied variants of PTC is detailed in Table 3. TROP-2 staining pattern was demonstrated in $86.9 \% \quad(53 / 61)$ of classic variant, $72.7 \%$ (8/11) of follicular variant (fig. 3) and 100\% (2/2) of tall variant, with the majority of all variants being diffuse $(3+$ and 4+). The staining difference between classic and FVPTC was not statistically significant ( $\mathrm{p}$ value $=0.807$ ). Similarly there was no significant staining difference between classic and studied non classic variants ( $\mathrm{p}$ value $=0.823$ ).

The results of TROP-2 expression in thyroid surgical specimens showed $85.1 \%$ sensitivity for the presence of PTC with 95\% confidence interval (CI) of $75.1 \%$ to 91.7 percent. Specificity for absence of PTC was $94.4 \%(95 \%$ CI: $80.9 \%$ - 99.4\%), PPV was $96.9 \%$ (95\% CI: $88.8 \%$ to $99.8 \%$ ) and NPV was $75.6 \%$ (95\% CI: $61.2 \%$ to $85.9 \%$ )
Overall diagnostic accuracy was $88.1 \%$ (95\% CI: $80.7 \%$ to $93.1 \%)$.

\section{TROP-2 expression in cell blocks sections of FNAC:}

TROP-2 staining results of cytological cell blocks along with histopathological follow up were shown in Table 4. Among cytologically diagnosed PTC cases, 23 out of 25 cases $(92 \%)$ demonstrated TROP-2 positivity (fig.5 and fig. 6).

These 23 positive cases were proved to be PTC on resection. All suspicious cases demonstrated TROP-2 positivity. They were diagnosed as PTC on histopathologic followup. One out of $15 \mathrm{FN}$ cases (6.7\%) showed positivity. Histopathologically, it was Follicular adenoma. Of FLUS cases, 3 of $11(27.3 \%)$ demonstrated TROP-2 positivity. Two cases were proved to be PTC on histopathological follow-up while the remaining case was FA (fig.7). None of the nodular hyperplasia cases showed TROP-2 immunoreactivity. Expression of TROP 2 was increased significantly in PTC compared with other thyroid samples ( $\mathrm{p}$ value $<0.001)$

Regarding staining distribution of positive cytological samples, the majority of PTC cases showed 3+ and 4+, while the two positive FA cases revealed $1+$ (Table 5).

Table 4: TROP-2 expression in cytological samples related to histopathological diagnosis

\begin{tabular}{|c|c|c|c|c|c|c|c|c|c|c|}
\hline \multirow{3}{*}{ Cytological Diagnosis } & \multirow{3}{*}{$\begin{array}{c}\text { Total } \\
(n=66)\end{array}$} & \multicolumn{9}{|c|}{ Histological follow up } \\
\hline & & \multirow{2}{*}{$\begin{array}{c}\text { TROP-2 positive } \\
\text { No. }(\%)\end{array}$} & \multicolumn{2}{|c|}{ HN } & \multicolumn{2}{|c|}{ FA } & \multicolumn{2}{|c|}{ FC } & \multicolumn{2}{|c|}{ PTC } \\
\hline & & & -ve & $+\mathrm{ve}$ & -ve & + ve & $-v e$ & + ve & -ve & + ve \\
\hline PTC & 25 & $23(92)$ & 0 & 0 & 0 & 0 & 0 & 0 & 2 & 23 \\
\hline Suspicious & 5 & $5(100)$ & 0 & 0 & 0 & 0 & 0 & 0 & 0 & 5 \\
\hline FN & 15 & $1(6.7)$ & 2 & 0 & 11 & 1 & 1 & 0 & 0 & 0 \\
\hline FLUS & 11 & $3(27.3)$ & 0 & 0 & 8 & 1 & 0 & 0 & 0 & 2 \\
\hline Nodular hyperplasia & 10 & 0 & 10 & 0 & 0 & 0 & 0 & 0 & 0 & 0 \\
\hline
\end{tabular}

PTC: papillary thyroid carcinoma; FC: follicular carcinoma; FA: follicular adenoma; FN: follicular neoplasm; FLUS: follicular lesion of undetermined significance; HN: hyperplastic nodule; TROP-2: human trophoblast cell-surface marker. 
Table 5: TROP-2 staining distribution in positive cytological cell blocks materials.

\begin{tabular}{|c|c|c|c|c|c|c|}
\hline \multirow[t]{2}{*}{ Histopathologic Diagnosis } & \multirow[t]{2}{*}{ Total No. (66) } & \multirow[t]{2}{*}{ Positive No. (\%) } & \multicolumn{4}{|c|}{ Distribution score } \\
\hline & & & $1+$ & $2+$ & $3+$ & $4+$ \\
\hline PTC & 25 & 23 & 0 & 3 & 8 & 12 \\
\hline Suspicious & 5 & 5 & 0 & 0 & 0 & 5 \\
\hline FN & 15 & 1 & 1 & 0 & 0 & 0 \\
\hline FLUS & 11 & 3 & 1 & 0 & 2 & 0 \\
\hline Nodular goiter & 10 & 0 & 0 & 0 & 0 & 0 \\
\hline
\end{tabular}

PTC: papillary thyroid carcinoma; FN: follicular neoplasm; FLUS: follicular lesions of undetermined significance; TROP-2: human trophoblast cell-surface marker

The results of TROP-2 expression in thyroid FNAC showed a sensitivity of $93.8 \%$, specificity $94.1 \%$, PPV $93.8 \%$, and NPV $94.1 \%$ in identifying PTC. Overall diagnostic accuracy was 93.9 percent. The calculated $95 \%$ CI of the sensitivity was from $79.2 \%$ to $99.2 \%$, of specificity was from $80.3 \%$ to $99.3 \%$, of PPV was from $79.2 \%$ to $99.2 \%$, of NPV was from $80.3 \%$ to $99.3 \%$ and of overall diagnostic accuracy ranged from $85.2 \%$ to 98.3 percent.

\section{DISCUSSION}

PTC is the most prevalent type of malignant tumor of thyroid gland. The diagnosis of PTC is, usually but not always, easily achieved with almost minimal interobserver variability. ${ }^{16}$ However, some cases do raise controversy as being PTC or non PTC due to subtle PTC nuclear features. ${ }^{17}$ Accurate diagnosis of such cases is very critical for patients' management and incorrect interpretation can lead to significant psychological and social problems as well as unnecessary surgical interventions. ${ }^{7}$

In this respect, the role of IHC markers in the diagnosis of PTC cannot be underestimated and large efforts have been made to detect new reliable IHC markers for diagnosing and distinguishing PTC from other follicular thyroid lesions. ${ }^{8}$ The most frequently recommended markers were HBME-1, galectin-3, and CK19. However these markers have poor specificity as they stain benign lesions; in spite of no diffuse staining pattern was reported. Other limitations include varying degree of sensitivity, focal staining in equivocal lesions, aberrant staining in oncocytic tumors, and false positivity in Hashimoto's thyroiditis. These drawbacks limit their use in practice. ${ }^{16}$

Few recent studies have examined the role of TROP-2 in diagnosing PTC. ${ }^{6,11,12,15}$ However, there was no agreement on the scoring system or its exact positive cut-off value, since all studies used different methods for IHC evaluation (automated or visual assessment). Also they used different quality of antibodies (mono- or polyclonal antibody). Most of these studies investigated TROP-2 on tissue microarray specimens with only few utilizing whole slide sections.
Some of these authors recommended additional studies to be performed on cases from different institutions to confirm their preliminary results. ${ }^{6,11}$ Therefore, this study was planned to investigate the possible role of TROP-2 in diagnosing PTC on 110 histological specimens and 66 cytological cell block materials of PTC and other thyroid lesions with follicular pattern.

Our results revealed that PTC presented significantly with TROP-2 overexpression compared to other studied follicular lesions both in surgical pathology specimens and cytological material ( $\mathrm{p}$ value $<0.001$ ); suggesting the potential diagnostic role of TROP-2 in identifying papillary thyroid carcinoma. These findings were consistent with previous investigations which suggested that TROP-2 is a highly promising marker for PTC diagnosis. ${ }^{11,12,15}$ We could also suggest that diffuse TROP-2 expression represented a good supportive evidence for the diagnosis of papillary thyroid carcinoma

In the current study, among surgically excised thyroid nodules, $85.1 \%$ ( 63 of 74 ) of PTC cases revealed TROP-2 staining pattern, with most positive cases (48 of 63) being diffusely stained $(3+$ or $4+)$. In contrast, FA, FC, nodular hyperplasia and adjacent normal thyroid tissue lacked TROP-2 expression except in one FA case (1+) and one FC case (3+). All studied Hurthle cell adenoma were negative. TROP-2 expression in thyroid surgical specimens showed $85.1 \%$ sensitivity for the presence of PTC, $94.4 \%$ specificity for absence of Papillary Thyroid Carcinoma. To explore more about TROP-2 expression in PTC, TROP-2 staining was also studied on 66 cytological cell block materials. On the same line with our histopathological results, TROP-2 detected all PTC cases except two (false negative) and only two benign cases (false positive). Most PTC positive cases were diffusely stained $(3+$ or $4+)$ while the 2 positive FA cases were focally stained (1+). We reported that TROP2 expression had a sensitivity of $93.8 \%$ for detecting PTC and specificity of $94.1 \%$ for absence of papillary thyroid carcinoma.

Higher sensitivity and specificity for detecting PTC 


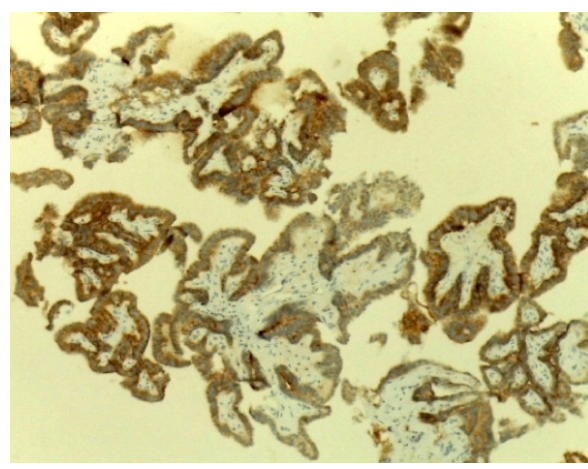

Figure 5: Cell block section of classic PTC reveals strong and diffuse positivity for TROP-2 (Immunoperoxidase; 100x).

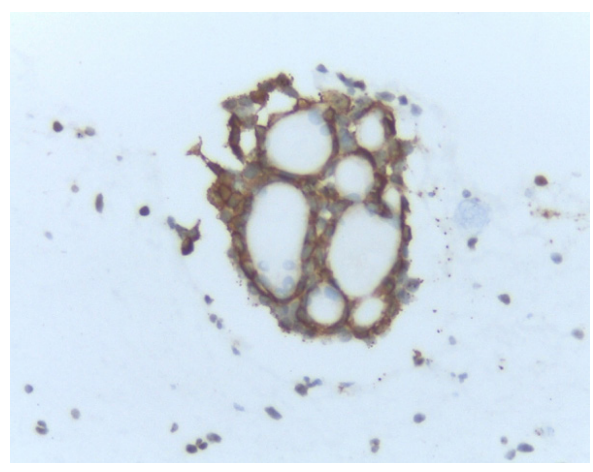

Figure 7: Cell block material of FA shows positivity for TROP-2 (Immunoperoxidase; X400).

(100\% for both) were documented in a previous study on TMA histpathological sections ${ }^{18}$; where TROP-2 was positive in all examined PTC cases and it was negative in all other studied thyroid lesions which include normal tissue, adenoma, Grave's disease, thyroiditis, Goiter, medullary carcinoma, clear cell carcinoma, squamous cell carcinoma, and undifferentiated carcinoma. They referred the lower sensitivity and specificity in other studies to usage less quality of TROP-2 antibody for IHC applications (polyclonal antibody).

Bychkov et al., in their work on excised thyroid sections using automated digital image analysis, recorded $82.5 \%$ TROP-2 positivity in PTC cases. ${ }^{6}$ They did not observe any staining in FA or FC cases and did not record background staining as well, so they reported that TROP-2 marker is superior over the most commonly used markers (CK19, galectin-3, and HBME-1) as it overcome their drawbacks of staining some negative cases and background. They explained the positivity in benign lesions in other studies either due to accidental positivity or aberrant Hurthe cell neoplasm staining due to high endogenous biotin activity.

Simms et al., on their work on TMA sections of thyroid neoplastic and non-neoplastic lesions, demonstrated 90\% $(54 / 60)$ TROP-2 positivity of their PTC cases. ${ }^{12}$ They also

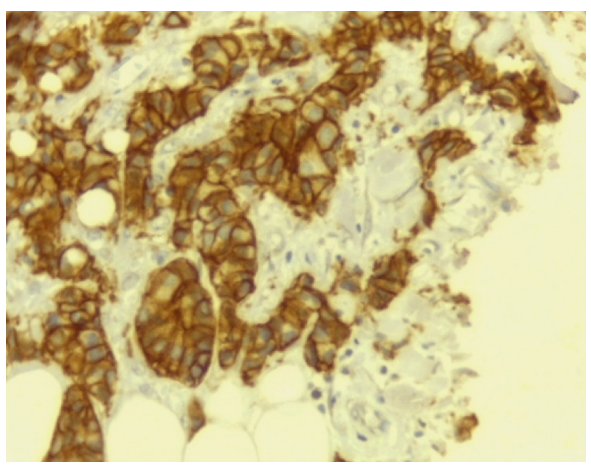

Figure 6: Cell block material of classic PTC shows strong and diffuse positivity for TROP-2 (Immunoperoxidase; X400).

noticed positivity in one of $27 \mathrm{FC}$ cases and 3 of 14 anaplastic carcinomas. In contrast, all their medullary carcinomas, FA and benign thyroid tissues did not demonstrate TROP-2 staining. Their results showed $90 \%$ sensitivity and $95.2 \%$ specificity. In their work on cell block cytologic materials, the authors revealed sensitivity of $95.3 \%$ and specificity $89 \%$, for detecting papillary thyroid carcinoma.

Nearly similar to our results, Addati et al., reported immunoreactivity in $8.3 \%(2 / 24)$ of FA cases. ${ }^{11}$ Sensitivity and specificity of $87 \%$ and $89 \%$, respectively were calculated for surgically excised sections. Sensitivity of $100 \%$ and specificity of $89 \%$ were recorded for their studied cytological materials as TROP-2 stained one FLUS case that proved to be FA on histological follow up. They demonstrated 91\% concordance between TROP-2 expression on histopathological sections and cytological materials. On comparing the TROP-2 results with HBME1 staining, they reported at least a similar level of TROP-2 sensitivity and specificity to HBME-1, in both cytology and histology, so they recommended its usage as an alternative, or in addition to HBME-1.

Others reported $90 \%$ and $70 \%$ TROP-2 staining in TMA preparations and whole surgical tissue sections of their studied PTC cases, respectively. ${ }^{15}$ On the other hand, none of their studied FA, FC (including Hurthle cell neoplasm), nodular hyperplasia, chronic lymphocytic thyroiditis and normal thyroid tissue showed staining. They explained the positivity difference in their study between TMA and whole sections to the difference in PTC variants where most TMA cases had classic morphology that showed strong positivity, while whole surgical sections had microcarcinoma and FVPTC with equivocal histomorphology and lower positivity. They also reported $100 \%$ TROP- 2 positivity on their examined cytology materials.

Regarding different PTC variants, FVPTC is histotype characterized by overlapping follicular and papillary features, which represents a frequent type of thyroid malignancy diagnosed as indeterminate category during 
diagnosis. ${ }^{12}$ In the current work, no significant TROP-2 staining difference among different studied variants was reported ( $\mathrm{p}$ value $=0.823$ ). Our data demonstrated that $86.9 \%(53 / 61)$ of classic variant PTC, $72.7 \%(8 / 11)$ of FVPTC and $100 \%(2 / 2)$ of tall variant expressed TROP-2 staining. These findings are nearly in line with others. ${ }^{11,15}$

Our results contradict that reported in an early study where Finley et al., assessed sensitivity and specificity for FVPTC diagnosis using several genes involved in thyroid carcinogenesis, including TROP- $2^{19}$; and they noted that FVPTC could represent an obstacle for reaching high TROP-2 sensitivity. Later, others reported TROP2 positivity in only $18.8 \%$ of FVPTC on TMA sections compared to $90 \%$ of classic variant. ${ }^{12}$ In the same line in more recent study, others recorded that less than $50 \%$ (47.4\%) of FVPTC on surgically excised sections were positive for TROP-2 staining and the majority of them were focally stained as compared to $100 \%$ of classic variant and papillary microcarcinoma, as well as $90 \%$ of tall columnar variant that were strongly positive for TROP- $2 .{ }^{6}$

This high difference regarding TROP-2 expression in FVPTC different studies can be attributed to number of studied cases, alternative approaches (TMA versus whole slide sections) or scoring methods (semiquantitative versus automation). Examination of whole slide sections can overcome tumor heterogeneity and expose TROP2 immunoreactive areas that are lost in TMA. Automated assessment overcomes the subjectivity and interobserver variability associated with visual observation. ${ }^{6}$ In accordance with these study Addati et al., reported that classic variant TROP-2 positivity were homogenous on surgical sections while in FVPTC was focal, thus the positive areas might be lost in TMA sections. ${ }^{11}$

In the present work, TROP-2 staining was predominantly mixed membranous and cytoplasmic pattern. This observation was reported by others. ${ }^{20}$ Shvartsur and Bonavida, reported that TROP-2 is expressed in the cytoplasm when cells become malignant and in some cases of cancer metastasis and recurrence. ${ }^{21}$ TROP-2 cytoplasmic positivity might be explained by cross reactivity with epithelial cell adhesion molecule EpCAM/TROP-1 that encoded by another gene of the same family (TACSTD1) and has a high similar sequence with TROP-2. ${ }^{20}$ EpCAM was expressed in differentiated thyroid carcinoma including FC, both in membrane and cytoplasm. This cross reactivity with EpCAM might also be explained TROP-2 positivity in some FC cases. $^{22}$

\section{CONCLUSION}

In conclusion, TROP-2 positivity was significantly higher in PTC cases compared to all other studied non PTC lesions both in surgical pathology specimens and cytological materials. Diffuse expression represented a good supportive evidence for the diagnosis of PTC. No significant staining difference among studied papillary thyroid carcinoma variants was observed. TROP-2 did not demonstrate expression in nodular hyperplasia and adjacent normal thyroid tissue. Thus, IHC detection of TROP-2 may be useful as a diagnostic aid to differentiate PTC from other follicular lesion. However, it is recommended to assess TROP-2 positivity on larger number of FA and FC cases which are the most important differential diagnosis for papillary thyroid carcinoma.

\section{Conflict of interests: None}

\section{REFERENCES}

1. Haugen BR, Alexander EK, Bible KC, et al. 2015 American Thyroid Association Management Guidelines for adult patients with thyroid nodules and differentiated thyroid cancer. Thyroid 2016;26:1-133. $\underline{\text { Crossref }}$

2. Majumder KR, Karmakar R, Karim SS, Al-Mamun A. Malignancy in solitary thyroid nodule. Mymensingh Med J 2016;25:39-44. Crossref

3. Keh SM, El-Shunnar SK, Palmer T, Ahsan SF. Incidence of malignancy in solitary thyroid nodules. J Laryngol Otol 2015;129:677-81. $\underline{\text { Crossref }}$

4. Palo S, Mishra D. Prevalence of malignancy in multinodular goiter and solitary thyroid nodule: a histopathological audit. Int J Res Med Sci 2016;4:2319-23. Crossref

5. Wu G, Wang J, Zhou Z, Li T, Tang F. Combined staining for immunohistochemical markers in the diagnosis of papillary thyroid carcinoma: Improvement in the sensitivity or specificity? J Int Med Res 2013;41:975-83. rossref

6. Bychkov A, Sampatanukul P, Shuangshoti S, Keelawat S. TROP-2 immunohistochemistry: a highly accurate method in the differential diagnosis of papillary thyroid carcinoma. Pathology 2016;48:425-33. Crossref

7. Liu H, Lin F. Application of Immunohistochemistry in Thyroid Pathology. Arch Pathol Lab Med 2015;139:67-82. Crossref

8. Alshenawy HA. Utility of immunohistochemical markers in differential diagnosis of follicular cell-derived thyroid lesions. Microsc Ultra 2014;2:127-36. $\underline{\text { Crossref }}$

9. Zeng P, Chen B, Zhou LN, Tang M, Liu PH, Lu PH. Impact of TROP2 expression on prognosis in solid tumors:A Systematic Review and Meta-analysis. Sci Rep 2016;20:33658. $\underline{\text { Crossref }}$

10. Trerotola M, Cantanelli P, Guerra E, et al. Upregulation of Trop-2 quantitatively stimulates human cancer growth. Oncogene 2013;10;32:222-33. Crossref 
11. Addati T, Achille G, Centrone M, et al. TROP-2 expression in papillary thyroid cancer: a preliminary cyto-histological study. Cytopathol 2015;26:303-11. Crossref

12. Simms A, Jacob RP, Cohen C, Siddiqui MT. TROP-2 Expression in Papillary Thyroid Carcinoma Potential Diagnostic Utility. Diagn Cytopathol 2016;44:26-31. $\underline{\text { Crossref }}$

13. Delellis RA, Lloyd RV, Heitz PU, Eng C, editors. Pathology and Genetics of Endocrine Organs: Tumors of the Thyroid and Parathyroid. In WHO Classification of Tumors. Third edition. Vol 3. Lyon; IARC Press 2004. pp. 49-73.

14. Theoharis CG, Schofield KM, Hammers L, Udelsman R, Chhieng DC. The Bethesda thyroid fine-needle aspiration classification system: year 1 at an academic institution. Thyroid 2009;19:1215-23. $\underline{\text { Crossref }}$

15. Liu H, Shi J, Lin F. The Potential Diagnostic Utility of TROP-2 in Thyroid Neoplasms. Appl Immunohistochem Mol Morphol 2016;9. [Epub ahead of print].

16. Zhang KE, Ge SJ, Lin XY, et al. Intercellular adhesion molecule 1 is a sensitive and diagnostically useful immunohistochemical marker of papillary thyroid carcinoma (PTC) and of PTC-like nuclear alteration in Hashimoto's thyroiditis. Oncol Lett 2016;11:1722-30. $\underline{\text { Crossref }}$
17. Kim YH, Yim H, Lee YH, et al. Evaluation of the VE1 antibody in thyroid cytology using Ex Vivo papillary thyroid carcinoma Specimens. J Pathol Transl Med 2016;50:58-66. Crossref

18. Liao Z. Immunohistochemical study of TROP-2 protein expression in thyroid lesions. [abstract]. In: Proceedings of the 107th Annual Meeting of the American Association for Cancer Research; 2016 Apr 16-20; New Orleans, LA. Philadelphia (PA): AACR; Cancer Res 2016;76(14 Suppl): Abstract pp 463.

19. Finley DJ, Arora N, Zhu B, Gallagher L, Fahey TJ 3rd. Molecular profiling distinguishes papillary carcinoma from benign thyroid nodules. J Clin Endocrinol Metab 2004;89:3214-23. Crossref

20. Stepan LP, Trueblood ES, Hale K, Babcook J, Borges L, Sutherland CL. Expression of Trop2 cell surface glycoprotein in normal and tumor tissues: potential implications as a cancer therapeutic target. $\mathrm{J}$ Histochem Cytochem 2011;59:701-10. Crossref

21. Shvartsur A, Bonavida B Trop2 andits overexpression in cancers: regulation and clinical/therapeutic implications. Genes Cancer 2015;6:84-105. $\underline{\text { Crossref }}$

22. Ensinger C, Kremser R, Prommegger R, Spizzo G, Schmid KW. EpCAM overexpression in thyroid carcinomas: a histopathological study of 121 cases. J Immunother. 2006;29:569-73. Crossref 